\title{
A dental care pathway for children with inherited bleeding disorders
}

Franchesca Fong*, Janet Davies, Janice Fearne, John Pasi

The Royal London Hospital is a major referral centre for children with inherited bleeding disorders (IBD). Dental caries and periodontal disease can be prevented, which is especially important in these children to avoid invasive treatment. For this reason a care pathway has been established, focusing on appropriate prevention advice and treatment

A Paediatric Dental Specialist attends the monthly Paediatric Haematology clinics. Children are screened for untreated dental decay and preventive dental advice is given verbally along with a patient information leaflet. At the clinic, a letter is sent out to the patient's general dental practitioner (GDP). Nonregistered patients are directed to NHS Choices website to find a local NHS GDP. Liaison of GDPs with both haematology and hospital paediatric dental services is actively encouraged to support the provision of dental care within the primary care setting, particularly routine preventive care. Depending on the severity of the bleeding diathesis and the degree of invasive dental treatment required, the GDP may undertake simple treatment or, in more complex cases, may arrange a referral to the Royal London Dental Hospital.

A consultation process takes place between paediatric dentist, paediatric haematologist and specialist nurse to determine the most appropriate haematological cover for each patient. This will depend on the severity of the bleeding disorder, the complexity of dental treatment and the need for local anaesthesia. The date of the dental visits and the haemostatic cover are requested via the electronic patient record so that it is accessible to all clinicians involved in their care without the need to retrieve their paper notes.

This pathway encourages active involvement of the patients' GDP and allows the patient to be treated as safely as possible in a timely manner. The care pathway has helped to formalise dental treatment for children with IBD and to improve every health care professional's understanding of their role in this care.

Key words: dental, haemophilia, inherited bleeding disorders, care pathway, paediatric, children

\footnotetext{
* Franchesca Fong, Paediatric Dental Department, Royal London Dental Hospital, New Road, London. Email: franchesca.fonganhs.net
}

Janet Davies, Paediatric Dental Department, Barts Health NHS Trust, London, UK

Janice Fearne, Paediatric Dental Department, Barts Health NHS Trust, London, UK

John Pasi, The Royal London Haemophilia Centre, Bart Health NHS Trust, London, UK

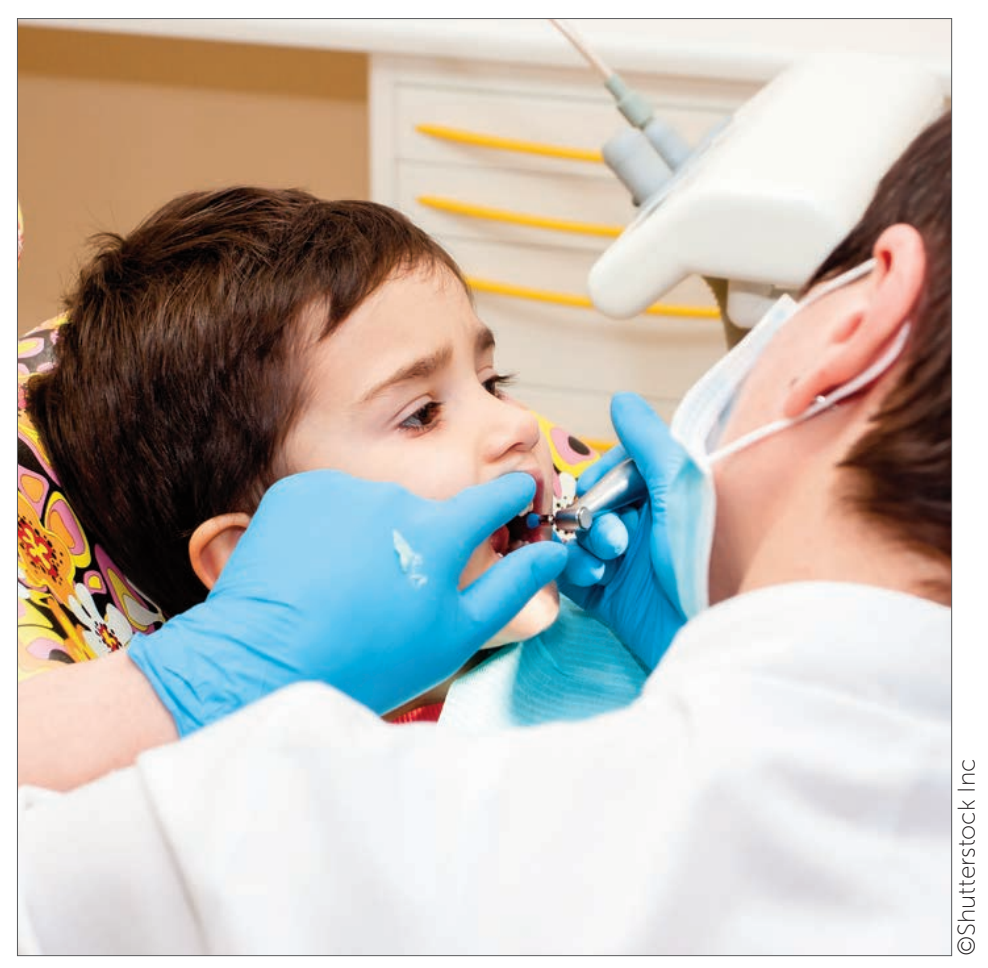

The Royal London Hospital is a major referral centre for over 250 children with inherited bleeding disorders (IBD) covering East and North East London and parts of Essex. Dental disease can place a further burden on these children and their parents, particularly if teeth require extraction. Dental treatment in patients with IBD can be complicated due to the potential for prolonged dental bleeding following invasive interventions. Children and parents can struggle to access primary dental care services; this could be related to a perceived lack of knowledge on the part of the general dental practitioner (GDP). In a survey of GDPs, approximately $50 \%$ were not confident with management of patients with IBD [1]. This can result in an increased risk of poor oral health in these patients and also a risk of untreated disease requiring a greater level of intervention than may have been needed if identified earlier. The presence of a dental specialist in the paediatric multidisciplinary haemophilia clinic helps to alleviate these risks by providing oral health advice, but a more coordinated approach is required.

The paediatric patient population in London has grown rapidly in recent years. According to the 2011 National Census, Tower Hamlets, the London Borough in which the Royal London Hospital is situated, had the highest population growth rate in England and Wales. The 


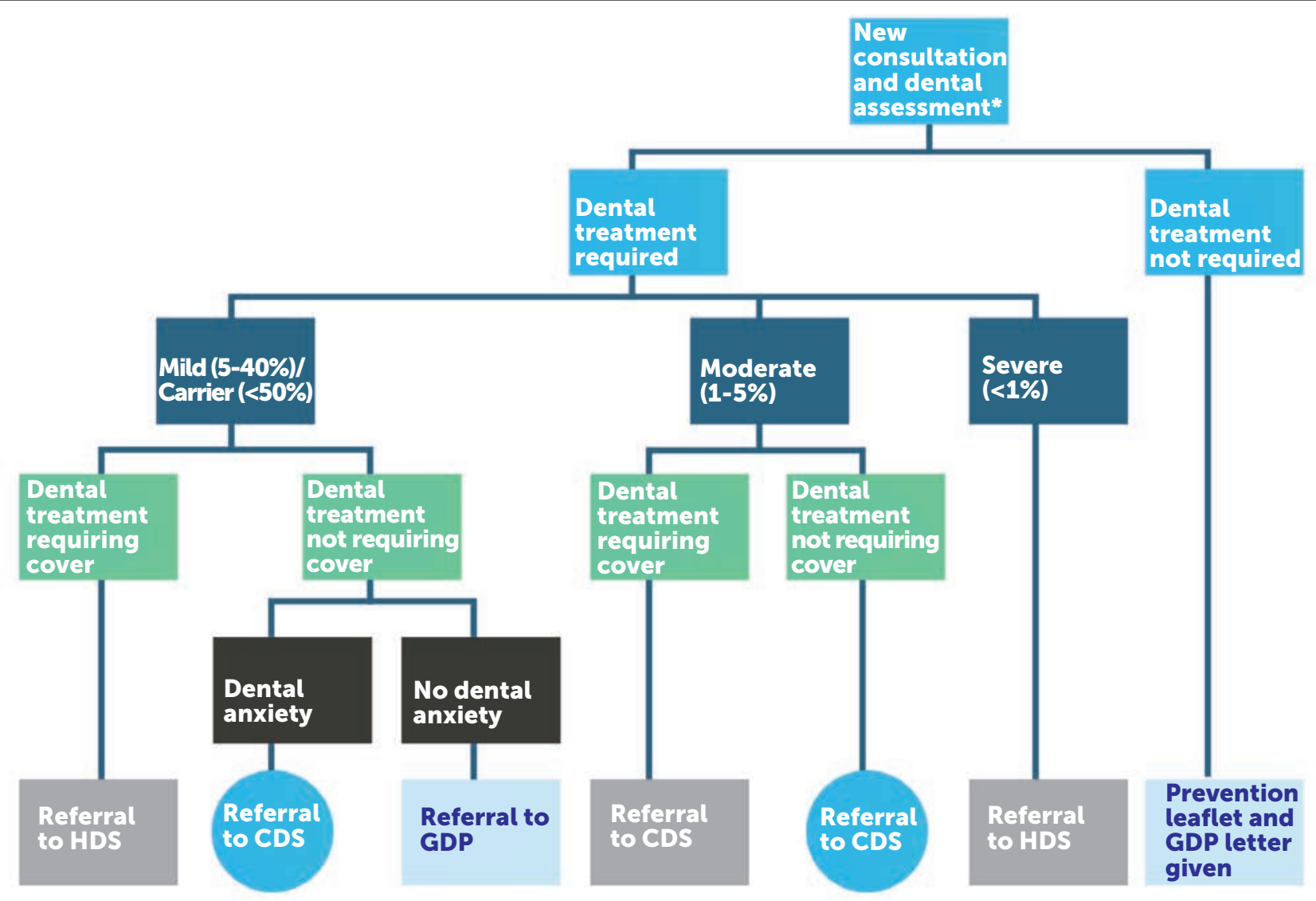

Figure 1: Paediatric Dental Care Pathway. * Non-registered patients are directed to NHS Choices to find a local NHS GDP if no active or urgent treatment is required. If imminent dental treatment is required, the patient will be taken on for one course of treatment within the HDS with emphasis on finding a GDP in the mean time. **Provided the haemophilia centre is in agreement

population of young children (aged 10 years and under) in the borough grew 19.2\% between 2001 and 2011 [2]. The area also has high levels of child poverty, which is linked to poorer oral health and increased risk of dental caries.

Furthermore, the introduction of a new dental contract in April 2006 led to a reduced availability of NHS primary dental care providers. Access to NHS primary dental services has become more challenging for the general public, leading to increased pressure on the Hospital Dental Service (HDS). This demonstrated a need to develop a care pathway for IBD patients that allows them to receive care from specialist paediatric dentists where appropriate, while GDPs provide the care that they feel confident to give. For adult patients, the Royal London already has a system of guided, shared care with the GDPs [1]. A new paediatric dental care pathway has, therefore, been established for similar reasons to try to focus our care on the patients that require specialist paediatric dental intervention in a hospital environment.

\section{Current guidelines}

In 2013, the United Kingdom Haemophilia Centres Doctors' Organisation (UKHCDO) dental working party published guidance on dental management of patients with IBD in both primary and secondary care [3]. These guidelines are mostly applicable to adults with some adaptation for paediatric patients, and state the following: - All local anaesthetic techniques including inferior dental block, buccal and lingual infiltration and intra-papillary and intra-ligamentary injections require cover.

- For moderate $(2-5 \%)$ to severe $(<1 \%)$ haemophilia patients, all dental treatment should be carried out in the HDS with a specialist dental unit, unless previously agreed with the haemophilia centre.

- Those with mild haemophilia (5-40\%) or carriers with $<50 \%$ factor can be treated in the primary care setting by their GDP or community dental service (CDS). However, they should also be seen every two years by the specialist dental team at the haemophilia centre.

These guidelines were incorporated into the paediatric dental care pathway.

\section{The new dental care pathway}

The need for a formalised dental care pathway for paediatric patients with IBD was identified following the development of the pathway for adult patients. This was important as the UKHCDO guidelines for children differ with respect to the use of local anaesthesia techniques [3] 


\section{Table 1: Oral health advice in patient information leaflet (based on Department of Health Guidance [4])}

\section{$0-3$ years of age}

- As soon as teeth emerge, they should be brushed twice daily using a fluoridated toothpaste. Brush last thing at night and on one other occasion.

\section{- Parents should brush or supervise toothbrushing.}

- Use fluoridated toothpaste containing no less than 1,000ppm fluoride. It is good practice to use only a smear of toothpaste.

- The frequency and amount of sugary food and drinks should be reduced.

- Sugar-free medicine is recommended.

- Sugar should not be added to weaning foods.

- Breast feeding provides the best nutrition for babies.

- From 6 months of age infants should be introduced to drinking from a free-flow cup, and from age 1 year feeding from a bottle should be discouraged.

\section{3-6 years of age}

- Brush at least twice daily, with a fluoridated toothpaste. Brush last thing at night and on one other occasion.

- Brushing should be supervised by a parent/carer.

- Use fluoridated toothpaste containing more than 1,000ppm fluoride. It is good practice to use only a pea-sized amount.

- The frequency and amount of sugary food and drinks should be reduced. Sugar-free medicine is recommended.

- Spit out after brushing and do not rinse, to maintain fluoride concentration levels.

\section{$>6$ years of age}

- Brush at least twice daily, with fluoridated toothpaste. Brush last thing at night and on at least one other occasion.

- Use fluoridated toothpaste $(1,350$ 1,500ppm fluoride)

- The frequency and amount of sugary food and drinks should be reduced.

- Sugar-free medicine is recommended.

- Spit after brushing and do not rinse, to maintain fluoride concentration levels. and, therefore, confusion could result. The care pathway was devised following discussion between all members of the haematology and paediatric dental teams and was based not only on the UKHCDO guideline but also on current practice and previous local experience (Figure 1). This proposed pathway aims to utilise primary dental care services such as the CDS and GDP for routine dental care that does not require cover so that the hospital services can manage the more complex treatments requiring cover.

Dental caries and periodontal disease are preventable oral diseases. As previously stated, some GDPs were not confident with the dental management of patients with IBD. They are, however, key players in the delivery of preventive dental advice and non invasive measures, such as applying fluoride varnish and fissure sealants. A specialist in paediatric dentistry attends the monthly paediatric haematology clinics. This individual determines whether or not the patient is registered with a GDP and provides the parents of those children who are not with details of how to register their child. In some cases where the child is particularly anxious or has other medical needs, the specialist may direct the parents to the CDS via the GDP as they are often more informed of the appropriate local CDS services. Preventive dental advice is provided both verbally and in the form of a patient information leaflet, which highlights the key points from the Department of Health (DoH) Delivering Better Oral Health guidance (Table 1) [4]. A letter is provided for the GDP/CDS, highlighting their role in prevention, explaining current treatment guidelines and providing contact details for the haemophilia team and specialist paediatric dentists. Liaison with GDPs by both the haematology and specialist dental services is actively encouraged not only to support provision of dental care within primary care, but also to allow GDPs to directly refer patients that need invasive dental treatment within the HDS.

The paediatric dental specialist also has a role in providing dental update seminars to the haemophilia team so that they can assist in providing the key oral health and preventive advice to children with IBD and their parents. Clinical nurse specialists will be in contact with children and parents before dentists become involved in their care and so have an invaluable opportunity to begin oral health promotion early in the child's life. Continuing professional development ensures that all members of the team are providing the same advice resulting in a clearer message to children and parents.

In cases that require cover and those with severe IBD who cannot be treated in primary care, the paediatric dental specialist organises their treatment within the HDS. A consultation process takes place between the paediatric haematologist, paediatric dentist and haemophilia clinical nurse specialist (CNS) to determine the most appropriate haematological cover. The cover provided will depend on the severity of the bleeding diathesis and the degree of invasive dental treatment required. Simple treatments such as fillings without local anaesthesia, supragingival scaling and provision of fissure sealants do not require cover. 
There are bleeding risks associated with provision of local anaesthetic, dental extractions, subgingival dental scaling and oral surgery in children. The haemophilia and dental team use an electronic messaging system to communicate dates, procedure details and the haemostatic cover to be provided. This message is directly linked to the electronic patient record so that it is accessible to all clinicians involved in their care without the need to retrieve paper notes. The haemophilia CNS will arrange the appropriate cover prior to their appointment and contact the parents to ensure they are aware of the need to attend the haemophilia centre prior to treatment. The CNS also organises any post-operative treatment that may be requried, such as tranexamic acid tablets or mouthwash.

\section{Discussion}

This multidisciplinary team approach allows for improved liaison between the members of the team, while combining expertise enables the provision of better care and treatment planning. A formalised care pathway allows safe and timely provision of dental care by primary care, the CDS and the HDS. The GDP is better informed of the patient's medical history and in their role in providing dental care. By providing direct contact details for both the haemophilia and specialist dental teams, the GDP can feel confident that they have access to advice as necessary. This is particularly important as guidelines differ for adults and children and confusion can arise, particularly with regard to the use of local anaesthetic. For adults, only inferior dental blocks and lingual infiltration require cover, whereas for children, all local anaesthetics require cover. Greater caution is needed in children as the consequences of uncontrolled bleeding are more serious.

An issue with both the guideline and the care pathway is that of transition from paediatric to adult health services, which usually occurs between 16 and 18 years of age. The adolescent patient with IBD may be safely treated as an adult accessing a greater proportion of invasive dental care from the GDP/CDS. Treatment provided local to the home is more convenient and may help to minimise absence from school for medical and dental care. For each individual, a structured consultation process that involves the child and their parents, both the GDP/CDS and specialist dentist and medical and nursing staff takes place to determine when they should transition into the adult service and whether an interim care pathway may be appropriate. Further research is required in this area.

Providing dental seminars to the haemophilia team aids in transferring key oral health and preventive advice for patients and parents with IBD. Development of a patient information leaflet is useful, but cannot be relied upon as parents may be unable to read or have a poor understanding of English. The use of health advocates is particularly important in such cases. It is also imperative that the advice given is as consistent as possible to reinforce positive preventive messages. The haemophilia team is kept updated of any changes in DoH oral health guidelines in oral health prevention. An interesting area for research would be the effect of early preventive interventions on caries rates in these patients. Dental hygienists and therapists could have an important role in treating these patients, particularly as they can now provide dental care through direct access without the need for a prescription from a dentist. This is another area in which useful research could be undertaken.

This pathway encourages active involvement of the patient's local dentist, which improves access to preventive dental care, increased local involvement and confidence of GDPs. This helps to provide a more patient-oriented service and to prevent dental problems, and allows the secondary HDS to focus on more complex and demanding cases. The care pathway needs to be audited in the future and adjusted, as necessary, in line with findings.

\section{References}

1. Kalsi H, Nanayakkara L, Pasi KJ, Bowles L, Hart P. Access to primary dental care for patients with inherited bleeding disorders. Haemophilia 2012; 18(4): 510-515 2. Tower Hamlets Council. 2011 Census Results: Headline Analysis. Population

growth in Tower Hamlets, UK; July 2012 .
http://www.towerhamlets.gov.uk/idoc.ashx?docid=3e6f8654-7214-4882-b548http://www.towerhamlets.go

3. Anderson JAM, Brewer A, Creagh D, Hook S, Mainwaring J, McKernan A, Yee TT, Yeung CA. Guidance on the dental management of patients with haemophilia and congenital bleeding disorders. British Dental Journal 2013; 215: 497-504

4. Department of Health \& The British Association for the Study of Community Dentistry. Delivering Better Oral Health: An evidence-based toolkit for prevention (Second Edition), GN2014126. London; 2014.

\title{
The Journal of Haemophilia Practice
}

\author{
An open-access journal for sharing \\ experience in the care of people \\ with bleeding disorders
}

www.haemjournal.com

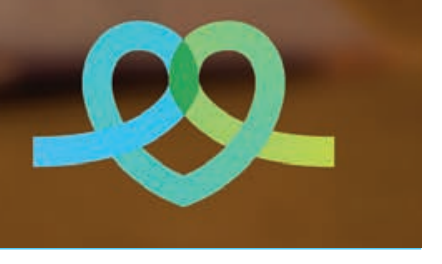

